\title{
The Learning Curve of Total Laparoscopic Hysterectomy in a Rural Hospital
}

\author{
Ismail BIYIK'ㄹ, Mustafa ALBAYRAK², Fatih KESKIN³, Ayse Nur USTURALI MUT ${ }^{4}$
}

ABSTRACT

OBJECTIVES: Online education and certification programs which help most gynecologic surgeons to advance, improve and prove their skills. However, the benefits of such distant programs in terms of complication rate and operation time has not been evaluated so far. The aim of this study was to report the improvement of a single surgeon's learning curve in total laparoscopic hysterectomy who had no previous mentorship/fellowship education, working in a rural district hospital before and after the completion of a distant on-line education and certification program - Gynaecological Endoscopic Surgical Education and Assessment.

STUDY DESIGN: Medical records of patients who underwent total laparoscopic hysterectomy between May 2015 and December 2018 were retrospectively reviewed and grouped based on the certification date of the surgeon, Group 1 before and Group 2 after certification. Groups were compared for variables that impact the learning curve (operation time, complications and conversion to laparotomy)

RESULTS: Of the 57 women eligible for evaluation 30 had total laparoscopic hysterectomy in Group 1 and 27 had total laparoscopic hysterectomy in Group 2. BMI, number of vaginal/cesarean births, previous abdominal/pelvic surgeries, operation indications, uterine weight, adnexectomy, and adhesiolysis rates, transfusion requirements, and the decrease in hemoglobin before and after operation were similar between the groups ( $p>0.05)$. Operation time was significantly shorter in Group 2 (83 min vs.116 min, $p<0.0001)$.

CONCLUSION: Thirty total laparoscopic hysterectomy operations seem enough to reach a plateau in the learning curve for gynecologists working in rural areas with limited facilities who cannot afford lengthily and expensive fellowship/mentorship programs, after completing distant online certification programs.

Keywords: Certification, Gynaecological endoscopic surgical education and assessment, Rural hospital, Total laparoscopic hysterectomy

\section{Gynecol Obstet Reprod Med 2020;26(2):134-139}

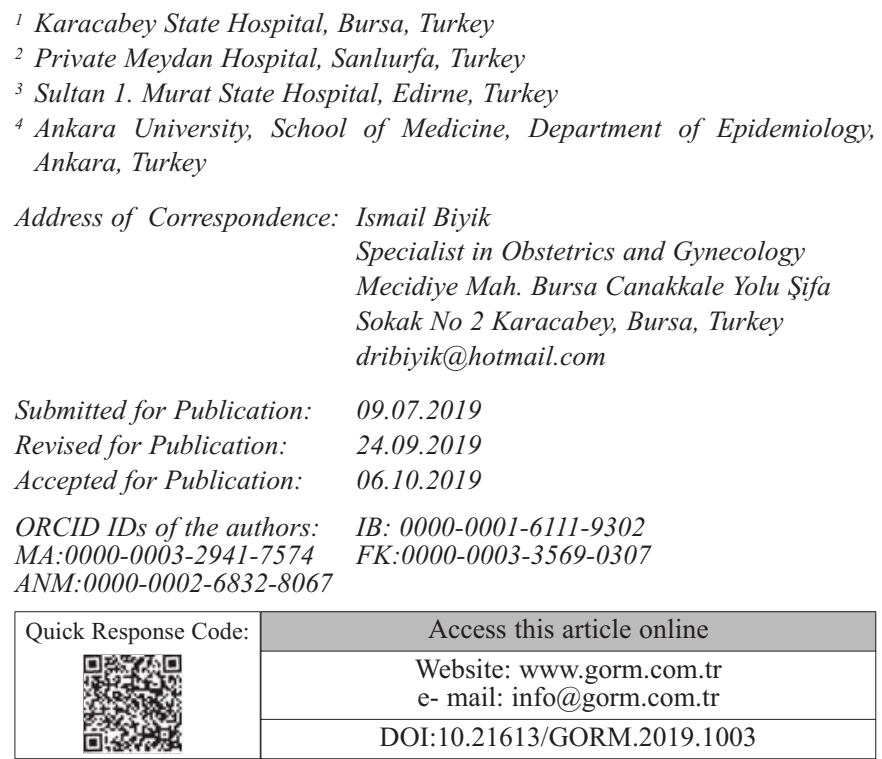

How to cite this article: Biyik I. Albayrak M. Keskin F. Usturali Mut AN. The Learning Curve of Total Laparoscopic Hysterectomy in a Rural Hospital. Gynecol Obstet Reprod Med 2019;25(0):00-00

\section{Introduction}

Hysterectomy for benign conditions is the most common gynecological operation performed globally (1). It can be done abdominally, vaginally or laparoscopically. The American Association of Gynecologic Laparoscopists (AAGL) recommends minimal invasive surgery whenever feasible (2). However, despite the advantages of the laparoscopic route such as less severe pain, bleeding, infection, postoperative adhesion formation, and shorter hospital stay, only $20 \%$ of hysterectomies are performed laparoscopically in USA (3-5). Besides the equipment cost and longer operation time, one obstacle for wider use of laparoscopy is the longer learning curve compared to abdominal hysterectomy $(5,6)$.

It is proven that operation times and complication rates decrease considerably after reaching a plateau in the learning curve and fellowship education programs are highly effective (7-9). However, it is hard for most gynecologists to be en- 
rolled in such rather lengthily and expensive programs. Instead, but not a one-to-one substitute for fellowship programs, is the on-line education and certification programs that help most gynecologic surgeons to advance, improve and prove their skills. However, as far as we know, the benefits of such distant programs in terms of complication rate and operation time has not been evaluated so far.

The aim of this study is to report the improvement of a single gynecological surgeon's learning curve in laparoscopic total hysterectomy (TLH) in a rural district hospital before and after the completion of a distant on-line learning and certification program-Gynaecological Endoscopic Surgical Education and Assessment (GESEA).

\section{Material and Method}

This was a retrospective study reviewing the medical records of patients who underwent TLH performed by a single gynecologic surgeon between May 2015 and December 2018 at Karcabey Hospital, a small general district hospital in Bursa province. Institutional review board approval was not obtained because of the retrospective nature of the study. The study was conducted in accordance with the Declaration of Helsinki. Permissions required for the study were taken. Only patients with simple hysterectomy (with or without removal of adnexa) were included. Patients with additional surgical procedures were excluded.

The data regarding the demographics, preoperative medical and surgical history (including previous pelvic surgeries, pelvic inflammatory disease, endometriosis), physical examination findings, indication for surgery, type of the procedure, total operating time, uterine weight, pathology reports, duration of hospital stay, and immediate intraoperative and postoperative complications were collected. Intraoperative complications included bleeding requiring transfusion; bladder, bowel, and ureteral injury; subcutaneous emphysema; and unintended conversion to abdominal hysterectomy. Postoperative complications were classified as 1) major: hemorrhage requiring treatment, fistula formation, thrombosis, embolism, or reoperation within 8 weeks or 2) minor: fever; infiltration of the vaginal vault (clinically palpable and painful mass or sonographically detected mass more than $4 \mathrm{~cm}$ without complaints); wound complications; and others (urinary tract infections, etc).

Gynecologist postgraduates are authorized and licensed for every kind of operation in the gynecological field by the ministry of health in Turkey. Likewise, there are no codes or regulations prohibiting the gynecologist from performing any kind of laparoscopic operation by stipulating the completion of any certification program.

The 1st author, IB, following the completion of the gynecologic specialization education program in 2012, was able to perform simple laparoscopic operations such as tubal ligation, salpingectomy, and ovarian cyst excision. After completing many national and international education programs certification programs, he assisted a colleague experienced in laparoscopic hysterectomy during 2015 which is followed by performing 10 laparoscopic hysterectomy operations under supervision. Then he started to perform advanced laparoscopic operations as a single surgeon.

The performance of the surgeon (İ.B.) regarding the main focuses of interests such as total operating time (beginning with the first skin incision and ending with the last closure of an incision), intraoperative complications and conversion to laparotomy before and after completion of online distant education program- GESEA were compared. GESEA is a structured online diploma program of ESGE (Europen Society of Gynecologic Endoscopy) for gynecological endoscopy composed of three steps. In the first step, a theoretical exam is taken following online training. In the second step, trainees are asked to perform practical skill training by using the GESEA tools during courses at the Academy, or in other GESEA Training or Diploma Centers, and during ESGE congresses or events for residents and inexperienced endoscopic surgeons. In the third step, the Bachelor practical exams at an accredited GESEA Diploma Centre are taken. GESEA Level 1 Bachelor certificate is granted following completion of all steps successfully. The surgeon in the present study (IB) obtained The European Academy of Gynecological Surgery Bachelor in Endoscopy Certificate and European Society of Gynecological Endoscopy ESGE Bachelor in Endoscopy Diploma in November 2017. Patients were divided into two groups based on the date of operation whether a patient is operated before (Group 1) or after (Group2) the granting of diploma.

Surgeons exclusion criteria included anesthetic contraindications for laparoscopic surgery, total uterine prolapse $>$ Grade 1 and pelvic relaxation requiring an additional vaginal or abdominal procedure, presumed extensive intraabdominal pelvic adhesions, estimated uterine weight $1000>\mathrm{g}$, patient with $\mathrm{BMI}>45$, more than 3 previous pelvic or abdominal surgery and history of pelvic radiotherapy.

\section{Surgical technique}

Informed consent was routinely obtained. Patients used a rectal enema the night before the hysterectomy. The surgical team was composed of four individuals: one attending surgeon, two assistants with one handling the camera and the other using a uterine manipulator and one scrub nurse. All patients received standard prophylactic $1 \mathrm{~g}$ cephalosporin or 600 $\mathrm{mg}$ of clindamycin IV in patients allergic to penicillin at the beginning of surgery. General anesthesia was administered via endotracheal intubation.

The patients were placed in a modified lithotomy position with the hips extended at $180^{\circ}$ and the knees flexed at nearly 
$90^{\circ}$. Pneumoperitoneum was generated using a Veress needle inserted through the umbilicus until the intra-abdominal pressure was $12 \mathrm{mmHg}$. A $10 \mathrm{~mm}$ trocar was inserted through the umbilicus for the camera. Three additional $5 \mathrm{~mm}$ ports with one placed $5 \mathrm{~cm}$ left of the umbilicus and the two others were placed $2 \mathrm{~cm}$ medial and superior to the anterior superior left iliac crest. In cases of previous midline vertical surgical scar, umbilical hernia repair or repeated 3 low transverse incision history Veress needle was inserted through Palmer's point. A RUMIC uterine manipulator with a Koh $\mathrm{Cup}^{\mathrm{TM}}$ colpotomizer (Cooper Surgical; Trumbull, Connecticut, US) was introduced vaginally at the beginning of the procedure. The hysterectomy was performed using a Ligasure ${ }^{\mathrm{TM}}$ Maryland Tip grasping and dissection instrument (Covidien; Dublin, Ireland) and monopolar energy modalities. All vascular pedicles were ligated and cut by using the Ligasure ${ }^{\mathrm{TM}}$. A circular vaginal incision was performed with monopolar coagulation. The uterus was removed from the abdomen through the vaginal cuff. If uterine morcellation was necessary, it was carried out apex was closed intracorporeally with interrupted single stitches, using absorbable suture (VICRYL ${ }^{\circledR}$ suture \#1 [JK-10]; Ethicon). We did not perform routine cystoscopy. The specimen was sent for pathological investigation. The patients' hemoglobin levels were checked preoperatively and on the second day after surgery. All patients were evaluated clinically and by ultrasonography on the day of discharge.

\section{Statistical analysis}

The average operating time was determined for each group. Outcome measures, such as hospital stay and conversion to laparotomy were recorded and evaluated for statistical significance. Statistical analysis was performed with Student's t-test or $\chi^{2}$ test. Trends for the length of surgery were assessed with univariate lineer regression. $\mathrm{R}$ values (correlation coefficients) and 95\% CI were calculated. Probability below 0.05 was considered statistically significant. Welch T-test was used for comparison with other studies in literature performed on the learning curve of TLH.

\section{Results}

There were 81 patients who had TLH during the study period. However, 24 patients were excluded because of the additional surgical procedures during TLH leaving 57 women eligible for evaluation. 30 of these had TLH before certification (Group 1) and 27 had TLH after certification of the surgeon (Group 2).

Demographic features such as age, weight, height, BMI, gravidity, parity, number of vaginal and cesarean births and previous abdominal/pelvic surgeries were similar between the groups (Table I).

Operation indications, adnexectomy and adhesiolysis rates during TLH did not significantly differ between the groups $(p>0.05)$ (Table II). Verres needle was introduced at Palmar point in three women in Group 1 (one for a previous umbilical hernia operation, one for previous abdominal myomectomy and the other had three previous low-segment transverse incision plus appendectomy). None had Palmer point entry in Group 2. We had one bladder injury in Group 1 during dissection of the bladder, which is diagnosed intraoperatively and repaired laparoscopically with double-layer sutures. She did well with no long-term sequel. Another patient had to be converted to laparotomy due to myoma and uterus weighed $620 \mathrm{~g}$.

There were no significant differences among the groups for transfusion requirement, preoperative and postoperative and change in hemoglobin, uterus weight, and the duration of hospital stay $(p>0.05)$. Operation time was significantly shorter in Group 2 (83 min vs. $116 \mathrm{~min}, p<0.0001$ ). The reason for the single transfusion in the study was preoperative hemoglobin of $8.5 \mathrm{~g} / \mathrm{dL}$.

Linear regression analysis shows that operation time got shorter as the numbers of patients are operated $(r=0.404$; $p=0.002$ ) (Figure 1).

Additionally, operation time is got longer as the uterine weight increased $(r=0.104 ; p \leq 0.001)$ (Figure 2).

Table I: The demographic features of patients.

\begin{tabular}{lccc}
\hline & $\begin{array}{c}\text { Group 1 } \\
(\mathrm{n}=30)\end{array}$ & $\begin{array}{c}\text { Group 2 } \\
(\mathrm{n}=27)\end{array}$ & $p$ \\
\hline Age & $48.03 \pm 5.34$ & $47.37 \pm 4.32$ & 0.611 \\
Weight & $76.20 \pm 15.39$ & $75.07 \pm 13.68$ & 0.773 \\
Height & $160.16 \pm 7.93$ & $157.22 \pm 7.97$ & 0.164 \\
BMl & $29.68 \pm 5.56$ & $30.49 \pm 5.86$ & 0.595 \\
Gravidity & $3.13 \pm 2.47$ & $3.44 \pm 2.42$ & 0.634 \\
Parity & $2.76 \pm 2.35$ & $2.81 \pm 1.30$ & 0.926 \\
Vaginal delivery & $2.50 \pm 2.31$ & $2.62 \pm 1.36$ & 0.801 \\
Cesarean history & $13.3 \%$ & $11.1 \%$ & 0.561 \\
Previous abdominal surgery & $16(53 \%)$ & $10(37 \%)$ & 0.280 \\
\hline BMI: Body Mass & & & \\
\hline
\end{tabular}

BMI: Body Mass Index 
Table II: Indications and operation features

\begin{tabular}{|c|c|c|c|c|}
\hline \multirow{6}{*}{ Indication } & & Group $1(n=30)$ & Group $2(n=27)$ & $p$ \\
\hline & AUB & $23(76.7 \%)$ & $18(66.7 \%)$ & \multirow{5}{*}{0.170} \\
\hline & Myoma uteri & $3(10 \%) 4$ & $(14.8 \%)$ & \\
\hline & CPP & 0 & $3(11.1 \%)$ & \\
\hline & Adnexal mass & 0 & $1(3.7 \%)$ & \\
\hline & $\mathrm{CIN}$ & $4(13.3 \%)$ & $1(3.7 \%)$ & \\
\hline \multirow{2}{*}{ TLH } & + adnexectomy & $28(93.3 \%)$ & $25(92.6 \%)$ & 0.913 \\
\hline & + adhesiolysis & $5(16.7 \%)$ & $3(11.1 \%)$ & 0.547 \\
\hline Veress insertion point & Umbilicus & $27(90 \%)$ & $27(100 \%)$ & \multirow{2}{*}{0.091} \\
\hline \multirow{3}{*}{ Complication } & Palmar's point & $3(10 \%)$ & 0 & \\
\hline & Bladder injury & $1(3.3 \%)$ & 0 & \multirow{2}{*}{0.393} \\
\hline & Conversion to laparatomy & $1(3.3 \%)$ & 0 & \\
\hline Operation duration (minutes) & & $116.33 \pm 33.34$ & $83.14 \pm 27.46$ & $<0.001^{*}$ \\
\hline Need for transfusion & & $1(3.3 \%)$ & 0 & 0.339 \\
\hline Preoperative hemoglobin (g/dL) & & $11.30 \pm 1.16$ & $1.06 \pm 1.38$ & 0.486 \\
\hline Postoperative hemoglobin ( $\mathrm{g} / \mathrm{dL})$ & & $10.20 \pm 1.19$ & $9.8 \pm 1.16$ & 0.208 \\
\hline Decline in hemoglobin $(\mathrm{g} / \mathrm{dL})$ & & $1.09 \pm 0.60$ & $1.27 \pm 0.63$ & 0.276 \\
\hline Uterus weight $(\mathrm{g})$ & & $245 \pm 175$ & $225 \pm 133$ & 0.623 \\
\hline Stay in hospital (day) & & $2.66 \pm 0.84$ & $2.48 \pm 0.70$ & 0.374 \\
\hline
\end{tabular}

AUB: Abnormal uterine bleeding, CPP: Chronic pelvic pain. CIN: Cervical intraepitelial neoplasia. TLH: Total laparoscopic hysterectomy, ${ }^{\star}$ statistically significant

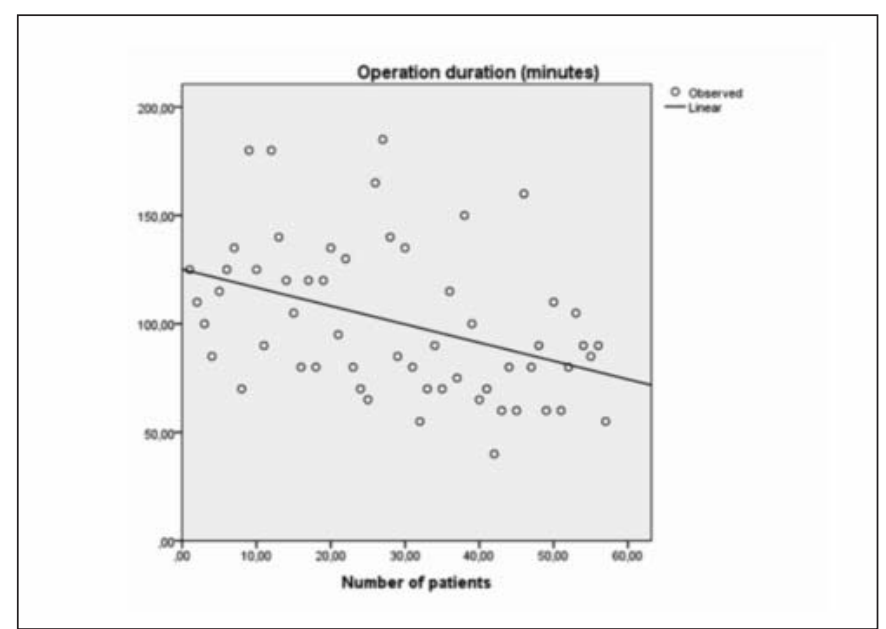

Figure 1: The linear regression analysis curve for the total operation time $(r=-0.404 ; p=0.002)$

\section{Discussion}

Advantages, safety and feasibility of TLH compared to abdominal hysterectomy are well established and it became a standard procedure in gynecologist's armamentarium in the last 30 years. However, only a competent surgeon who reached the plateau of the learning curve can serve the virtues of laparoscopy to his/her patients safely. Reaching a plateau in the learning curve and gaining competency in laparoscopic procedures is generally measured by the number of cases operated with a (1) decreased operation time with a (2) acceptable perioperative complication rate, and (3) conversion to laparotomy $(8,10,11)$. Despite the variables such as the difficulty of operations, motivation, and skill of the surgeon, pres-

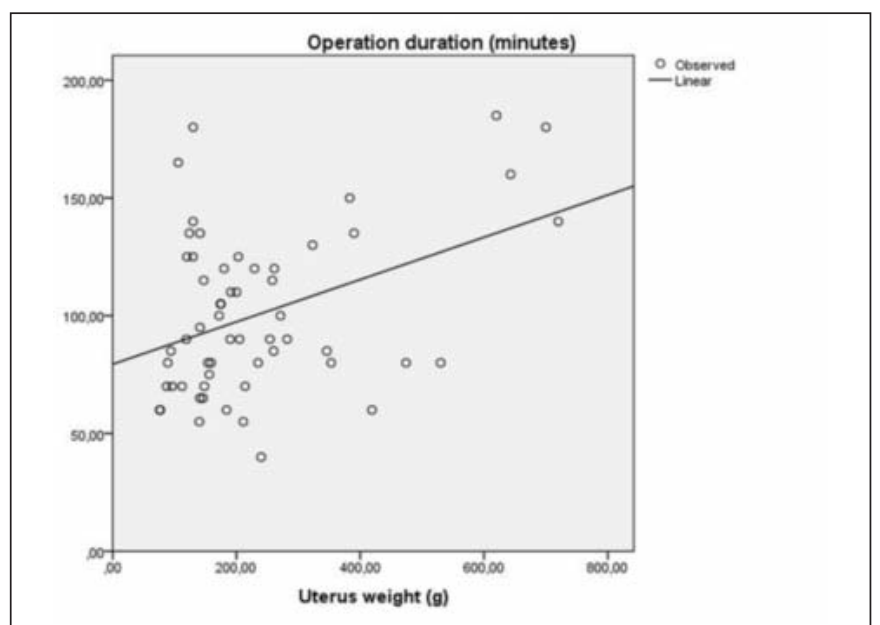

Figure 2: The linear regression analysis curve of uterus weight and total operation time $(r=0.104 ; p=<0.001)$

ence of supervision or mentorship, quality and expertise of assisting personnel and equipment quality defining the case number to complete the learning curve is important in the standardization of gynecologists' education. However, there is no standard case number agreed upon to reach a plateau in the learning curve for laparoscopic hysterectomy (Table III) $(8,10,12-15)$. Rosen et al. suggested as low as 16 cases in terms of duration of surgery for a trainee surgeon under supervision in a teaching hospital facility in which the operating time reduced from 180 minutes to 105 minutes (12). Twijnstra et al. concluded that 25 cases suffice under a mentorship program to reach a plateau of the learning curve in a teaching hospital (15). Mavrova et al. concluded that the operation time decreases after only 20 cases in TLH for inexperienced surgeons 


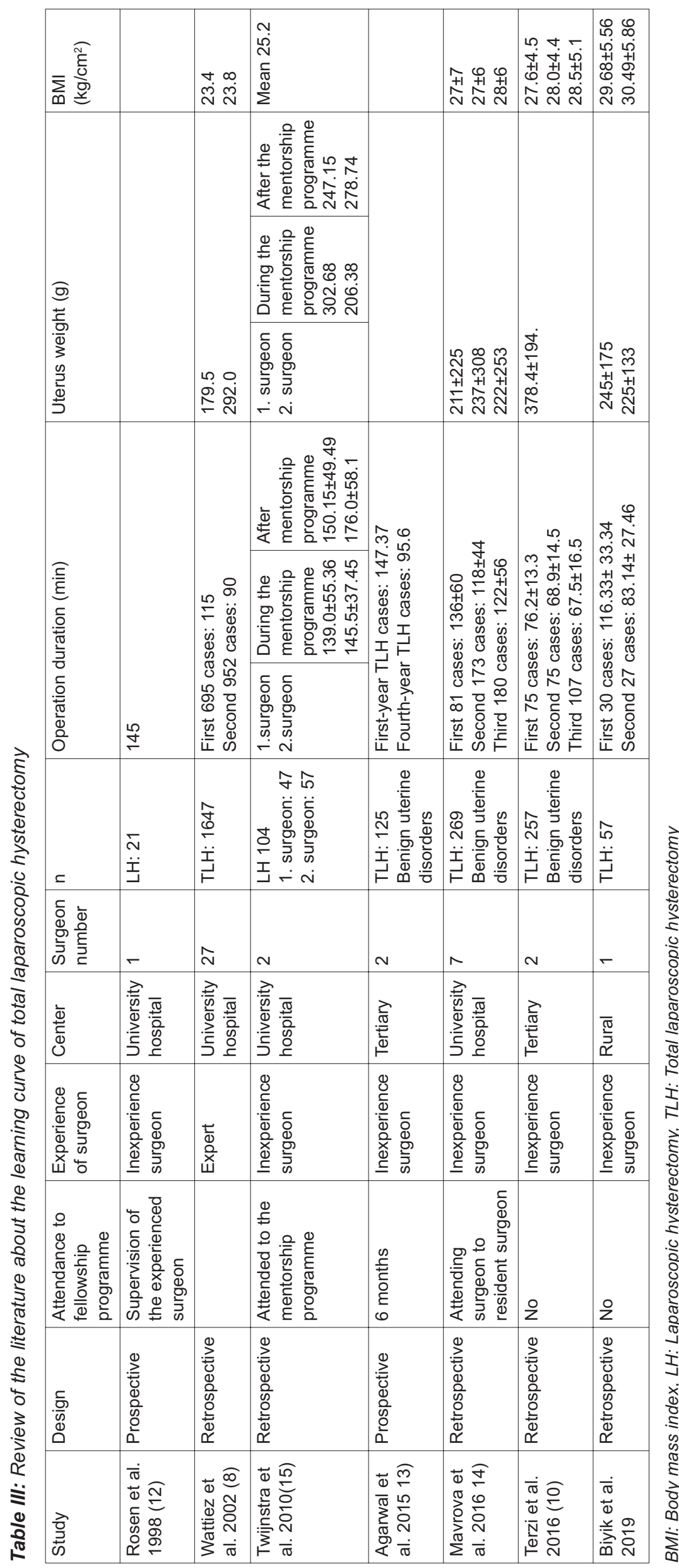

under mentorship with a decrease of the mean duration of surgery from $136 \mathrm{~min}$ to $118 \mathrm{~min}$. But additional procedures were included in TLH in some of their cases (14). However, Terzi et al. reported a longer learning curve in their retrospective series with two inexperienced gynecologists, up to 75 cases with shortening of operating time from 76 minutes to $68 \mathrm{~min}(10)$. They speculated that this might be linked to the selection of difficult cases, higher BMI, large uterine size and the absence of formal mentorship.

The significant decrease of operation time in our series from 116 min to $83 \mathrm{~min}$ after the first 30 cases following the granting of GESEA certification is comparable to previous studies. Our mean total operation time was even shorter than some of these publications despite the higher mean BMI of our patients (Table III). This may result from the patient selection criteria, previous experience of the surgeon in simpler laparoscopic operations and/or difference in surgical technique (We do not routinely perform ureter dissection or perform cystoscopy routinely). However, the uterine weight or the history of pelvic/abdominal surgery, which may create bias, is comparable to the aforementioned studies except for the uterine weight in Terzi et al (10).

The complication rate is another measure of reaching a plateau in the learning curve. The major complication rate for TLH is reported below $2 \%$ by experts $(8,16,17)$. However, this low complication rate may not be representative of inexperienced surgeons such as the gynecologists in the aforementioned studies discussed under decreased operation time. We are aware that we certainly cannot conclude that we reached the plateau of the learning curve in terms of complications since we had a small case number. Nevertheless, we had only one major complication in the study, which was in the first group. To note, we had also no major complication in the other 24 patients excluded from the study due to extra procedures in addition to TLH. The same may be concluded for the rate of conversion to laparotomy.

We think that the completion of the learning curve of a single surgeon (I.B.) in a rural province state hospital with limited facilities is the differentiating feature of the present study because of the previous reports on the learning curve of TLH were conducted either in a university or teaching hospital setting. And most had followed a struc- 
tured fellowship or mentorship program. The surgeon (I.B.) followed a distant learning GESEA Bachelor in Endoscopy Certificate program, which included theoretical, video-visual, training box and auditing studies after performing a few years of basic - simpler laparoscopic operations such as ectopic pregnancy and ovarian cystectomy. He had no structured mentorship or attended a fellowship program. We do not mean to underrate a mentorship/fellowship program which is obviously more advantageous but for those who cannot afford time and money, an online distant course may significantly contribute to the education of surgeons in rural areas.

Another distinctive feature of the present study is that it reflects the performance of a single surgeon. Most of the previous reports included at least two-beginner surgeons. Examining the performance single surgeon may help to exclude the confounding factors such as the skill of the surgeon. We also excluded patients with laparoscopic-assisted, laparoscopic subtotal hysterectomies in addition to patients with additional procedures, which helps to homogenize the results. To the best of our knowledge, our study is the first to report the learning curve of a single surgeon in a rural facility after an online distant education program.

\section{Conclusion}

Thirty TLH operations for benign cases is enough to reach a plateau in the learning curve for motivated gynecologists working in rural areas with limited facilities who cannot afford time and money for lengthy and expensive fellowship/ mentorship programs, especially after completing distant certification programs provided that surgeon is aware of his own limitations and selects convenient cases. We assume this is important for wider use and benefitting our patients from the virtues of TLH in rural areas instead of referral to tertiary centers.

\section{Acknowledgment: None}

Disclosure: No authors have any conflict of interest report. Funding: No

Author contribution: IB: Protocol/project development, data collection, manuscript writing/editing; MA: Manuscript writing/editing, supervision; FK: Data analysis, manuscript writing/editing; ANM: Data analysis and manuscript writing/editing

\section{References}

1. Garry R. The future of hysterectomy. BJOG. 2005;112 (2): 133-9.

2. AAGL Advancing Minimally Invasive Gynecology Worldwide. AAGL position statement: route of hysterectomy to treat benign uterine disease. J Minim Invasive Gynecol. 2011;18(1):1-3.

3. Cohen SL, Vitonis AF, Einarsson JI. Updated hysterectomy surveillance: factors associated with minimal invasive hysterectomy. JSLS. 2014;18(3). pii: e2014.00096.
4. Johnson N, Barlow D, Lethaby A, Tavender E, Curr L, Garry R. Methods of hysterectomy: systematic review and meta-analysis of randomised controlled trials. BMJ. 2005; 330(7506):1478.

5. Nieboer TE, Johnson N, Lethaby A, Tavender E, Curr E, Garry R, et al. Surgical approach to hysterectomy for benign gynaecological disease. Cochrane Database Syst Rev. 2009;(3):CD003677.

6. Bijen CB, Vermeulen KM, Mourits MJ, de Bock GH. Costs and effects of abdominal versus laparoscopic hysterectomy: systematic review of controlled trials. PLoS One. 2009;4(10):e7340.

7. Subramonian K, Muir G. The "learning curve" in surgery: what is it, how do we measure it and can we influence it? BJU Int. 2004;93(9):1173-4.

8. Wattiez A, Soriano D, Cohen SB, Nervo P, Canis M, Botchorishvili R, et al. The learning curve of total laparoscopic hysterectomy: comparative analysis of 1647 cases. J Am Assoc Gynecol Laparosc. 2002;9(3):339-45.

9. Clark NV, Gujral HS, Wright KN. Impact of a fellowshiptrained minimally invasive gynecologic surgeon on patient outcomes. JSLS. 2017;21(3). pii: e2017.00037.

10. Terzi H, Biler A, Demirtas O, Guler OT, Peker N, Kale A. Total laparoscopic hysterectomy: Analysis of the surgical learning curve in benign conditions. Int J Surg. 2016;35: $51-7$.

11. Garrett A.J, Nascimento MC, Nicklin JL, Perrin LC, Obermair A. Total laparoscopic hysterectomy: the Brisbane learning curve. Aust N Z J Obstet Gynaecol. 2007;47(1):65-9.

12. Rosen DMB, Cario GM, Carlton MA, Lam AM, Chapman M. An assessment of the learning curve for laparoscopic and total laparoscopic hysterectomy. Gynaecological Endoscopy. 1998;7(6):289-93.

13. Agarwal P, Bindal N, Yadav R. Risks and benefits of total laparoscopic hysterectomy and the effect of learning curve on them. J Obstet Gynaecol India. 2016;66(5):379-84.

14. Mavrova R, Radosa JC, Wagenpfeil G, Hamza A, Solomayer EF, Juhasz-Böss I. Learning curves for laparoscopic hysterectomy after implementation of minimally invasive surgery. Int J Gynaecol Obstet. 2016;134(2):225-30.

15. Twijnstra AR, Blikkendaal MD, Kolkman W, Smeets MJ, Rhemrev JP, Jansen FW. Implementation of laparoscopic hysterectomy: maintenance of skills after a mentorship program. Gynecol Obstet Invest. 2010;70(3):173-8.

16. Donnez O, Donnez J. A series of 400 laparoscopic hysterectomies for benign disease: a single centre, single surgeon prospective study of complications confirming previous retrospective study. BJOG. 2010;117(6):752-5.

17. Karaman Y, Bingol B, Günenç Z. Prevention of complications in laparoscopic hysterectomy: experience with 1120 cases performed by a single surgeon. J Minim Invasive Gynecol. 2007;14(1):78-84. 